Traditional Medicine and Modern Medicine

Vol. 1, No. 1 (2018) 59-67

(C) Institutes of Integrative Medicine, Fudan University and

World Century Publishing Corporation

DOI: $10.1142 / \mathrm{S} 2575900018500052$

\title{
Feixian Recipe inhibits pulmonary fibrosis by targeting pulmonary microvascular endothelial cells and VEGF/VEGFR2 signaling pathway
}

\author{
Lihong Meng ${ }^{1,2}$, Chen Wang ${ }^{1}$, Zijuan Wang ${ }^{1,2}$, Ting Yin $^{2}$, Zhe Liu ${ }^{1,2}$, Huihui Qin ${ }^{1}$, Yuting Zhang ${ }^{1,2}$, \\ Xiaofeng $\mathrm{Gu}^{1,2}$, Xiaolin $\mathrm{Yu}^{1,2}$, Liangduo Jiang ${ }^{2}$ and Xiaomei Zhang ${ }^{2, *}$ \\ ${ }^{1}$ Second Clinical Medical School, Beijing University of Chinese Medicine \\ Beijing 100029, P. R. China \\ ${ }^{2}$ Department of Respiratory Medicine \\ Dongfang Hospital Affiliated to Beijing University of Chinese Medicine \\ Beijing 100078, P. R. China \\ *Zhangxim6767@sina.com
}

Received 20 October 2017; Accepted 10 December 2017; Published 7 February 2018

\begin{abstract}
Objective: To investigate the regulatory mechanism of PMVECs and vascular endothelial growth factor VEGF/ vascular endothelial growth factor receptor 2 (VEGFR2) signaling pathway in pulmonary fibrosis and the inhibitory effect of Feixian Recipe (FXR) in pulmonary fibrosis by targeting VEGF/VEGFR2 signal pathway.
\end{abstract}

\begin{abstract}
Methods: In this study, pulmonary microvascular endothelial cells (PMVECs) were successfully isolated from rats with pulmonary fibrosis. Cells were divided into six groups: model group, prednisone group, losartan group and three different concentrated $(100 \mathrm{ug} / \mathrm{mL}, 60 \mathrm{ug} / \mathrm{mL}, 20 \mathrm{ug} / \mathrm{mL})$ FXR groups. The adhesion rate, migration and closed blood vessels of each PMVECs group were detected. The mRNA expression of VEGF, VEGFR2, phosphoinositide 3-kinase (PI3K), mitogen-activated protein kinases 38 (P38 MAPK) and activin receptor-like kinase (ALK) were detected by SYBR Green I real-time fluorescence quantitative PCR.
\end{abstract}

Results: Compared with the model group, the adhesion rate, migration and angiogenesis of PMVECs were decreased in FXR groups $(P<0.05)$. Compared with prednisone and losartan groups, the mRNA expressions of VEGF, VEGFR2, PI3K and P38 MAPK were down-regulated significantly by FXR $(P<0.05)$.

Conclution: FXR can inhibit the migration, adhesion and angiogenesis of PMVECs in rats with pulmonary fibrosis by targeting VEGF/VEGFR2 signal pathway, and inhibit the progress of pulmonary fibrosis.

Keywords: Feixian Recipe (FXR); pulmonary fibrosis; pulmonary microvascular endothelial cells (PMVECs); VEGF/VEGFR2 signaling pathway.

\section{Introduction}

Pulmonary fibrosis is a life-threatening disease, with unexplained causes, characterized by chronic inflammatory response and pulmonary interstitial fiber over-deposition. ${ }^{1}$ With the development of pulmonary fibrosis, the lung structure is destroyed, then it will result in respiratory failure and finally leads to death. In recent years, the pathogenesis of pulmonary fibrosis has been studied widely; the center has been transferred from inflammation theory to injury repair theory. ${ }^{2}$
It is considered that pulmonary microvascular angiogenesis plays an important role in the pathogenesis of pulmonary fibrosis. $^{3-5}$ Many studies have found that angiogenesis is an important pathological part of pulmonary interstitial fibrosis. $^{6,7}$ Adhesion, differentiation, proliferation and migration of pulmonary microvascular endothelial cells (PMVECs) play an important role for angiogenesis. ${ }^{8}$ In the area of pulmonary fibrosis, pulmonary microvessels increase significantly, and the course of angiogenesis

\footnotetext{
*Corresponding author.
} 
and inflammatory response and fibrosis repair is synchronous. ${ }^{9}$

Vascular endothelial growth factor (VEGF) is a key regulator of angiogenesis, and it plays a role mainly through the combination with its receptor. VEGF and vascular endothelial growth factor regulator (VEGFR2) binding is the main signaling pathway of vascular endothelial cell-mediated angiogenesis. ${ }^{10,11}$ Under physiological circumstances, a bit amount of VEGF expression can maintain vascular integrity, under pathological conditions, overexpressing VEGF will inhibit vascular endothelial cell apoptosis, promotes its proliferation, migration and differentiation, promotes angiogenesis, finally increases vascular permeability, leading to lung tissue abnormalities repair and extracellular matrix deposition. ${ }^{6}$

Feixian Recipe (FXR) is an effective prescription of idiopathic pulmonary fibrosis summarized from clinical treatments, with the benefit of replenishing and restoring lung and kidney, dissipating phlegm and eliminating blood stasis, and promoting blood circulation for removing obstruction in collaterals. This study will further explore the effect and mechanism of FXR on migration, adhesion and angiogenesis of PMVECs based on VEGF/VEGFR2 signal pathway, and investigate the effect of FXR on the mRNA expression of VEGF, VEGFR2, phosphoinositide 3-kinase (PI3K), mitogenactivated protien kinases 38 (P38 MAPK) and activin receptor-like kinase (ALK).

\section{Materials and Methods}

\section{Cell lines and cell culture}

Primary PMVECs were derived from Bleomycin-induced pulmonary fibrosis rats. Rats were provided by Beijing Weitong Lihua Animal Company (Beijing, China). PMVECs lines were established from rat lung tissues. The 28-day-old pulmonary fibrosis rats models were selected. After intraperitoneal injection of anesthesia, lungs were taken sterilely. Cut the tissues with a width of $2 \mathrm{~mm}$ on the edge of the lungs, then cut them into $1 \mathrm{~mm}^{3}$ size pieces, and put pieces into DMEM. Then, $2 \mathrm{~h}$ later, the cells were cultivated with DMEM containing $20 \%$ fetal bovine serum, and incubated in the $\mathrm{CO}_{2}$ incubator. Then, $60 \mathrm{~h}$ later, remove the tissue blocks and replace the culture medium, until the cells converged into a single layer, then cryopreserve them for the following experiments. The cells were divided into six groups: model group, prednisone group, losartan group, and high-, mid-, low-dose $(100 \mathrm{ug} / \mathrm{mL}, 60 \mathrm{ug} / \mathrm{mL}, 20 \mathrm{ug} / \mathrm{mL})$ of FXR groups.

\section{Drugs and chemicals}

FXR consists of Dongchong xiacao (cordyceps sinensis), Hongjingtian (rhodiola), Huangqi (astragalus), Yinxin (ginkgo), Gualou (trichosanthes), Haigeqiao (clam shell), Mahuang (ephedra) and Shuizhi (leech). FXR granules, each gram medicamental pulverized equivalent to $3.56 \mathrm{~g}$ dried medicinal herb, were provided by the Chinese Crude Drug Laboratory in the Institute of Economic and Resource Management of the Beijing Normal University (Beijing, China). Prednisone was $5 \mathrm{mg}$ per tablet, provided by the Tianjin Lisheng Pharmaceutical Co. Ltd. (China), product number: 1303041. Losartan tablets: $50 \mathrm{mg}$ per tablet, Hangzhou Merck East Pharmaceutical Co. Ltd. (China), product number: 120411. DAB coloring liquid, Bi Yuntian Cat. No. P0202. Trypsin, Rui Erkang Cat. No. REK3012. Matrigel glue, BD Cat. No. 356234. Goat anti-rabbit IgG $(\mathrm{H}+\mathrm{L})$-HRP, CWbio. Co. Ltd. Cat. No. CW0103. Anti-VIII antibody, ANNEXIN. DNase I CWbio. Co. Ltd., HiFi-MMLV cDNA first chain synthesis kit, CWbio. Co. Ltd., UltraSYBR Mixture (with ROX) CWbio. Co. Ltd. Transwell plates, Coring Cat. No. 3464.

\section{Measurement of cell migration}

Cell migration was assessed by Transwell plates, with $6.5 \mathrm{~mm}$ diameter and $8 \mu \mathrm{m}$ pore filters. PMVECs were divided into six groups: model group treated with DMEM containing $20 \%$ fetal bovine serum; high-dose, mid-dose and low-dose of FXR group treated with $20 \%$ fetal bovine serum and different concentrations ( $100 \mathrm{ug} / \mathrm{mL}, 60 \mathrm{ug} / \mathrm{mL}, 20 \mathrm{ug} / \mathrm{mL})$ FXR extract, respectively, losartan group treated with DMEM containing $20 \%$ fetal bovine serum and $5 \mathrm{ug} / \mathrm{mL}$ losartan, prednisone group treated with DMEM containing 20\% fetal bovine serum and $5 \mathrm{ug} / \mathrm{mL}$ prednisone. Equal numbers of PMVECs $\left(1 \times 10^{6} / \mathrm{mL}\right)$ were added to Transwell plates for each group. After $24 \mathrm{~h}$, discard the culture medium in the wells, wash the cells twice with phosphate-buffered saline (PBS), and then fix with methanol for $20 \mathrm{~min}$. After $0.1 \%$ crystal violet staining for 15-20 min, wash with water more than three times and using a cotton swab, wipe the upper layer of non-migratory cells gently. Use the $100 \times$ microscope to take pictures and then count the piercing cells.

\section{Adhesion of PMVECs detected by WTS-1}

About $2 \mathrm{~h}$ before assay, the original medium was replaced by $100 \mu \mathrm{L}$ serum-free medium and $10 \mu \mathrm{L}$ WST-1 solution. Then $2 \mathrm{~h}$ later, plate is shaked horizontally, and then the absorbance was read at $450 \mathrm{~nm}$. The adhesion rate of PMVECs was then detected.

\section{Angiogenesis experiment}

About $200 \mathrm{~mL}$ of Matrigel glue was paved in the 24-well plates. Group cells are digested with trypsin, suspended in the $0.1 \%$ serum DMEM and counted. Cells are diluted to a 
Table 1. RT-PCR detection of the target gene primer sequence.

\begin{tabular}{lcc}
\hline Gene & Primer $\left(5^{\prime}-3^{\prime}\right)$ & Post-primer $\left(5^{\prime}-3^{\prime}\right)$ \\
\hline PI3K & ACAAAGATGCTCTCCTGAACTGGCT & ATGCCAAGGACATAAGAGGCTACAC \\
ALK & GACAGACCCAACTTTGCATCATT & CACTTTCTCCTCCTCCTCTACTACGG \\
P38MAPK & GTACCTGGTGACCCATCTC & GATTATGTCAGCCGAGTGTAT \\
VEGF & GAGACCCTGGTGGACATCTT & GATCCGCATGATCTGCATAG \\
VEGFR2 & AAGAGATTTGTTCCGGATGG & CGGCAGATAGCTCAATTTCA \\
GAPDH & TGGAGTCTACTGGCGTCTT & TGTCATATTTCTCGTGGTTCA \\
\hline
\end{tabular}

density of $1 \times 109 / \mathrm{mL}$, then added $500 \mu \mathrm{L}$ of cell suspension per well. After the incubation for $18 \mathrm{~h}$, the number of closed blood vessels was recorded in the entire field under a $100 \times$ microscope and photographed.

\section{mRNA analysis by real-time polymerase chain reaction ( $R T-P C R)$}

Total RNA from the PMVECs was extracted using the TRIzol reagent and cDNA was synthesized using the Prime Script ${ }^{\circledR}$ RT reagent kit with gDNA Eraser, according to the manufacturer's instructions. RT-PCR was performed on the cDNA samples using the SYBR ${ }^{\circledR}$ Premix Ex TapTM II. The used primers are listed in Table 1 . The reaction conditions were as follows: $95^{\circ} \mathrm{C}$ for $30 \mathrm{~s}$, followed by 40 cycles of denaturation at $95^{\circ} \mathrm{C}$ for $5 \mathrm{~s}$ and extension at $60^{\circ} \mathrm{C}$ for $34 \mathrm{~s}$. All amplifications were done in triplicate and repeated three times. The PCR data were analyzed by the QuantStudio 7 Flex detection system. Cycle threshold (CT) values were analyzed by the comparative CT ( $\Delta \Delta \mathrm{CT})$ method, and the relative amount of target mRNA $\left(2^{-\Delta \Delta C T}\right)$ was obtained by normalizing to endogenous glyceraldehyde 3-phosphate dehydrogenase (GAPDH).

\section{Statistical analysis}

The data are expressed as mean \pm standard deviation (SD). They were analyzed with SPSS 20.0 (SPSS Inc., Chicago, IL,

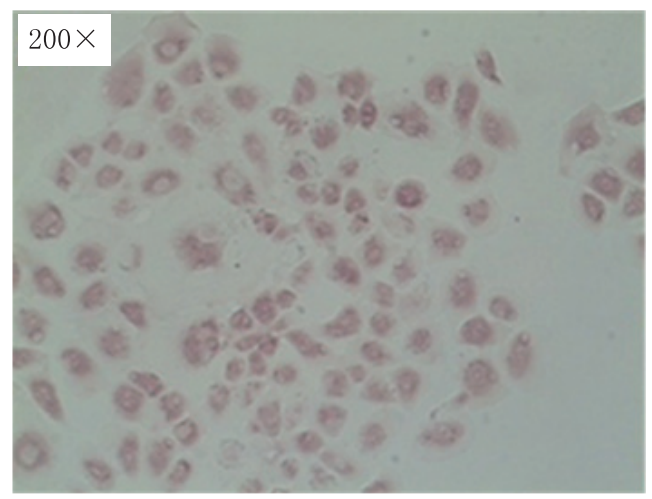

(a)
USA). One-way analysis of variance was performed to test the differences between the groups. $P<0.05$ was considered to be statistically significant.

\section{Result \\ Primary culture and identification of PMVECS}

The first passage of PMVECs from pulmonary fibrosis rats cultured in the present study had the typical morphological characteristics of microvascular endothelial cells, as distributed like paving stone typically and no overlapping growth phenomenon (Figs. 1(a) and 1(b)). The results of antigen immunohistochemistry of endothelial cell factor VIII released the positive expression of factor VIII protein in the cytoplasm, indicating that the cells were identified as vascular endothelial cells.

\section{FXR inhibited migration of PMVECs}

The inhibitory effect of FXR on PMVECs migration was measured by the Transwell assay (Fig. 2). Though prednisone group increased its migration, all other groups could inhibit the migration of cells. Compared with model group, highdose of FXR, mid-dose of FXR, and losartan group could inhibit the migration of cells significantly $(P<0.01)$. Compared with prednisone group, FXR and losartan group had significant inhibition of cells migration $(P<0.05)$.

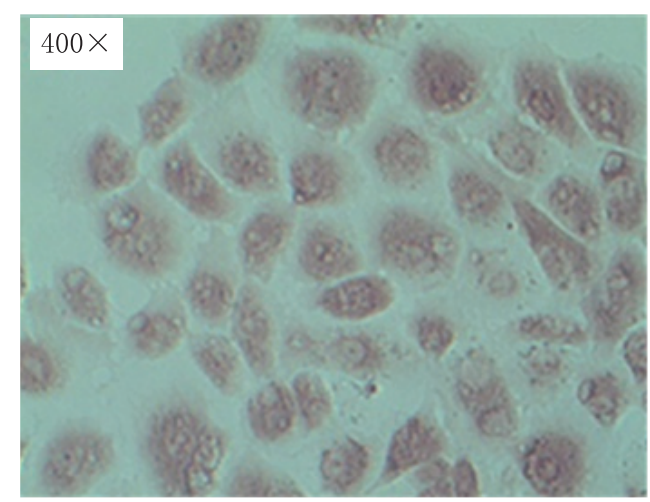

(b)

Figure 1. Cell source identification with immunohistochemistry assay shows that they are PMVECs: (a) Factor VIII protein was expressed positive in the cytoplasm, and cells distributed like paving stone typically and no overlapping growth phenomenon indicating that the cells were PMVECs (x200) and (b) (x400). 


\section{PMVECs}

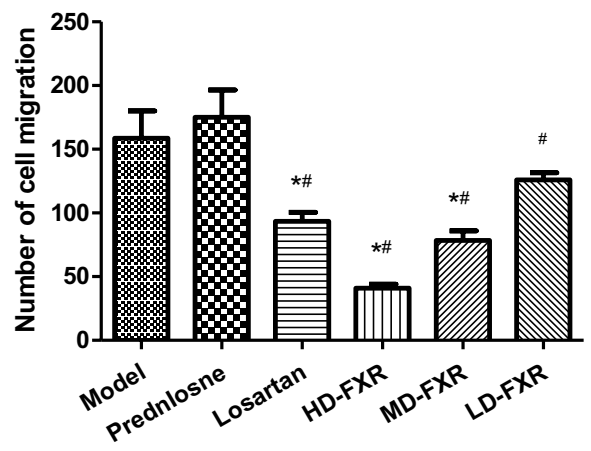

(A)

(a)

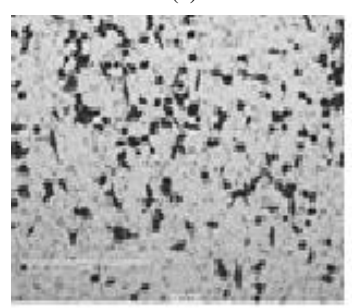

(d)

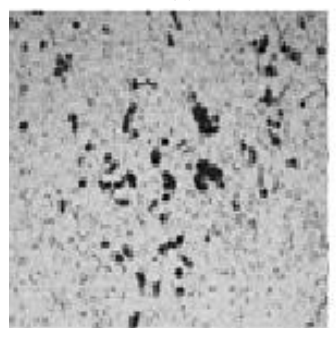

(b)

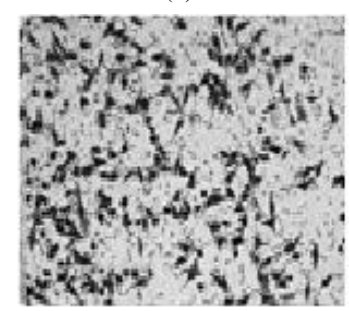

(e)

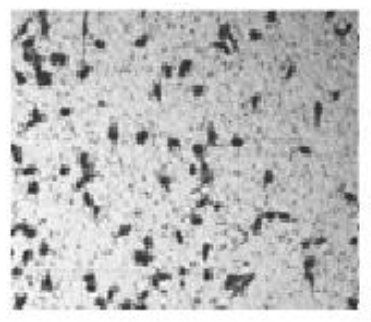

(c)

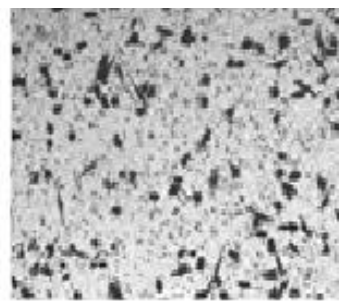

(f)

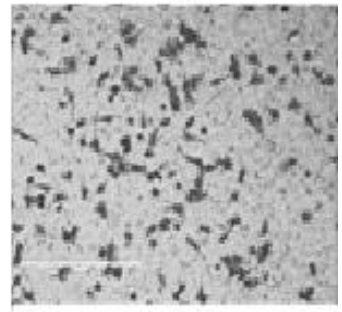

(B)

Figure 2. PMVECs treated with FXR, losartan and prednisone show altered migration after $24 \mathrm{~h}$ of treatment. The inhibitory effect of FXR on PMVECs migration was measured by the Transwell assay: (A) Quantitative analysis of the migrated PMVECs through the pores. (B) Representative images of the (a) model group, the (b) prednisone group, the (c) losartan group, the (d) high-dose of FXR group, the (e) mid-dose of FXR group and the (f) low-dose of FXR group. Values are expressed as the mean $\pm \mathrm{SD}(n=3)$.

Notes: ${ }^{*} P<0.01$, compared with the model group; ${ }^{\#} P<0.05$, compared with the prednisone group. Model: model group, Prednisone: prednisone group, Losartan: losartan group, HD-FXR: high-dose of FXR group, MD-FXR: mid-dose of FXR group, LD-FXR: low-dose of FXR group.

\section{FXR inhibited the adhesion of PMVECs}

The adhesion rate and average optical density (OD) value of PMVECs were detected by WST- 1 at 12, 24 and $48 \mathrm{~h}$, respectively (Fig. 3). There was no significant difference in the adhesion of cells among all groups at $12 \mathrm{~h}$. FXR and losartan group can reduce adhesion rate of PMVECs at 24 and $48 \mathrm{~h}$. Figures 3B(b) and $3 \mathrm{~B}(\mathrm{c})$ show that the high-dose of FXR and losartan group down-regulate the OD value of PMVECs significantly compared with model group at 24 and $48 \mathrm{~h}(P<0.05)$.

\section{Effect of FXR on inhibiting angiogenesis in PMVECS}

Different doses of FXR and losartan groups could reduce the neovascularization significantly $(P<0.05)$. With the dose of
FXR increased, the inhibitory effect of angiogenesis was enhanced. High-dose of FXR and losartan group almost inhibited angiogenesis completely.

\section{Effect of FXR on RNA expression of VEGF and VEGFR in PMVECs}

After treatment with different concentrations of FXR, losartan and prednisone for $24 \mathrm{~h}$, the RNA expression levels of VEGF and VEGFR2 were detected by SYBR Green I RT fluorescence quantitative PCR. Figure 5(a) shows that FXR can reduce the RNA expression of VEGF, and it is more significance than the losartan and prednisone $(P<0.05)$. Figure 5(b) shows that losartan and prednisone can decrease 


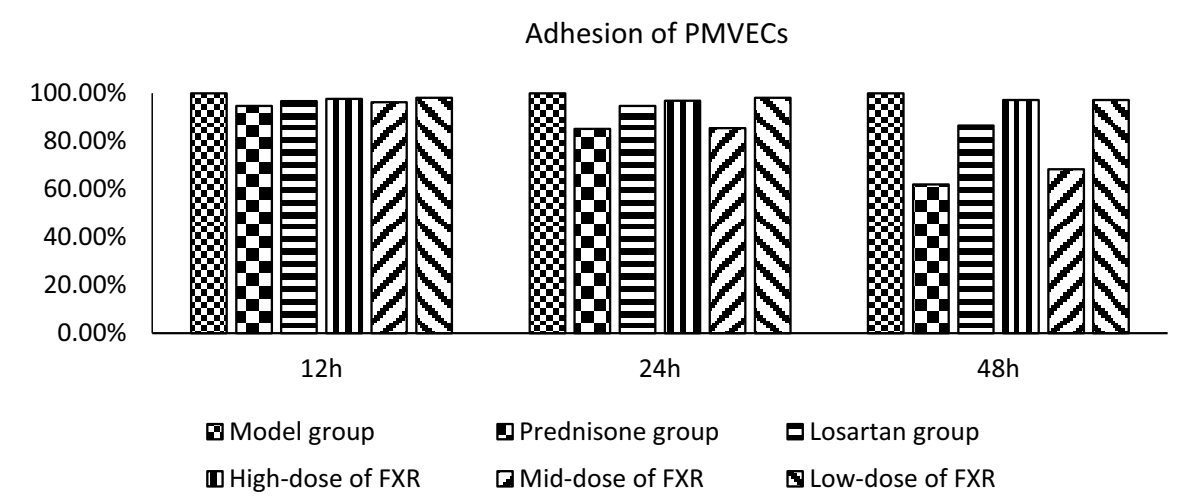

(A)

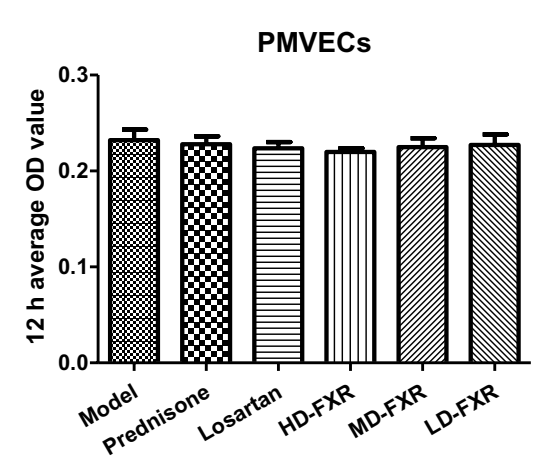

(a)

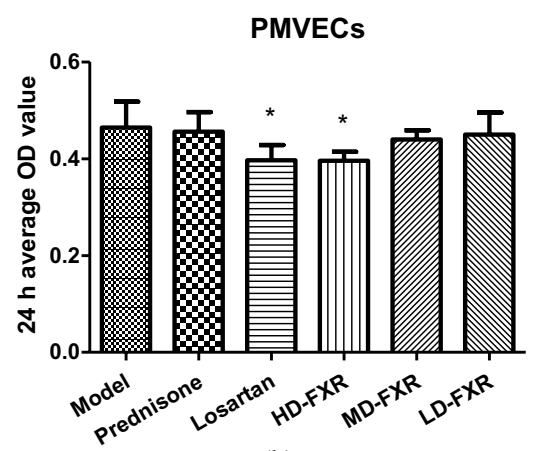

(b)

PMVECs

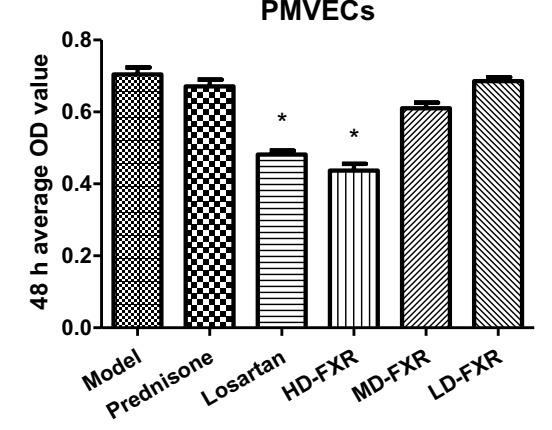

(c)

(B)

Figure 3. FXR treatment inhibited the adhesion rate of PMVECs: (A) The adhesion rate of PMVECs was detected by WST-1 at 12,24 and 48 h, respectively. (B) Colorimetric assay of the absorbance at $450 \mathrm{~nm}$ for each group at (a) 12 , (b) 24 and (c) $48 \mathrm{~h}$, respectively. Values are expressed as the mean $\pm \mathrm{SD}$ ( $n=3$ ).

Notes: ${ }^{*} P<0.05$, compared with the model group. $n=6$ independent experiments. Model: model group, Prednisone: prednisone group, Losartan: losartan group, HD-FXR: high-dose of FXR group, MD-FXR: mid-dose of FXR group, LD-FXR: low-dose of FXR group.

the RNA expression of VEGFR, and have more advantage than FXR $(P<0.05)$.

\section{Effect of FXR on RNA expression of PI3K, P38MAPK and ALK in PMVECs}

After treatment with different concentrations of FXR, losartan and prednisone for $24 \mathrm{~h}$, the RNA expression levels of PI3K, P38MAPK and ALK were detected by SYBR Green I RT fluorescence quantitative PCR. Figures 6(a) and 6(b) show that FXR, losartan and prednisone can down-regulate the RNA expression of PI3K and P38MAPK $(P<0.05)$. Figure 6(c) shows the RNA expression of ALK in FXR, losartan and prednisone groups are up-regulated than model group, but high-dose of FXR can inhibit the upward trend.

\section{Discussion}

Many experiments had demonstrated that the vascular overgrowth, inflammatory response and fibrosis repair occurred at 


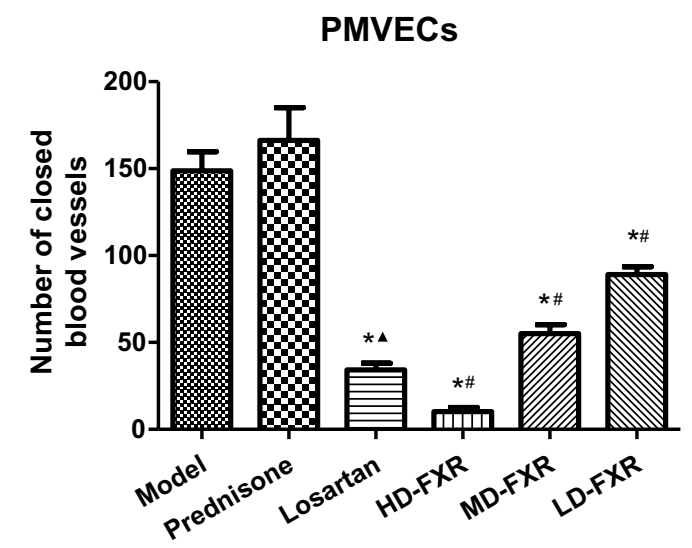

(A)

(a)

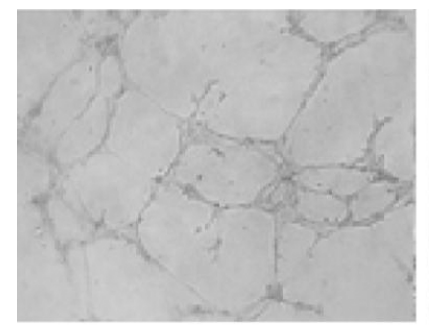

(d)

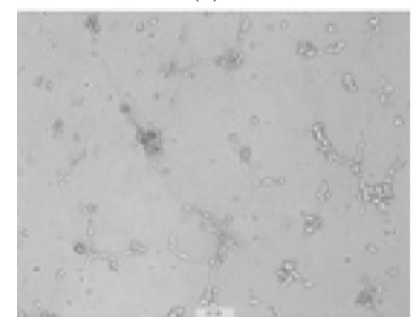

(b)

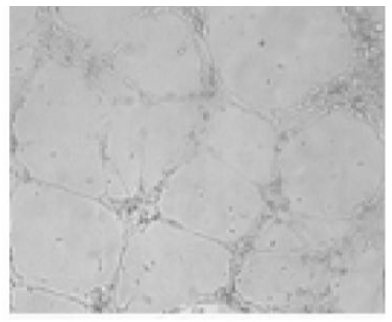

(e)

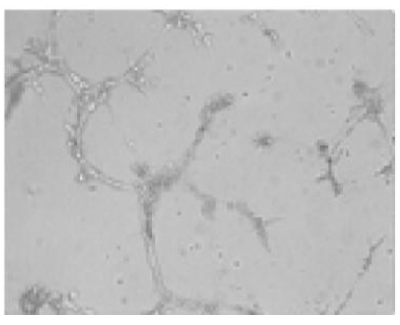

(B) (c)

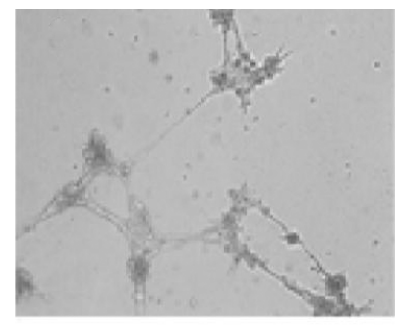

(f)

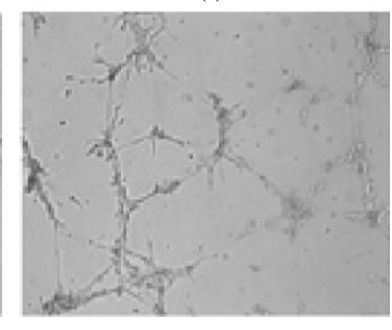

Figure 4. Inhibitory effect of FXR on angiogenesis of PMVECs: (A) Quantitative analysis of angiogenesis of PMVECs after treated with FXR, losartan and prednisone. (B) Representative images of the (a) model group, the (b) prednisone group, the (c) losartan group, the (d) high-dose of FXR group, the (e) mid-dose of FXR group and the (f) low-dose of FXR group. Values are expressed as the mean $\pm \operatorname{SD}(n=3)$.

Notes: $* P<0.05$, Compared with the model group and prednisone group; ${ }^{*} P<0.05$, three FXR groups compared with each other. $\mathbf{\Delta} P<0.05$, compared with the low-dose of FXR group. $n=3$ independent experiments. Model: model group, Prednisone: prednisone group, Losartan: losartan group, HD-FXR: high-dose of FXR group, MD-FXR: mid-dose of FXR group, LD-FXR: low-dose of FXR group.

the same time in pulmonary fibrosis. When the pulmonary inflammatory response occurred in the early pulmonary fibrosis animal model, we observed the pulmonary vascular endothelial damage and proliferation, converted from the anti-adhesion, anticoagulation, diastolic phenotype to promote adhesion, coagulation, contraction phenotype, followed by the microvascular endothelial cells which are migrated and proliferated. ${ }^{12,13}$ After extensive lung injury among pulmonary fibrosis, abnormal angiogenesis is the start and push factor of fibrosis repair and fibrous deposition. VEGF is the main signaling molecule that mediates angiogenesis. VEGF/VEGFR2 is the main pathway of vascular endothelial cell-mediated angiogenesis, and it can also activate many other factors that have promoting function on pulmonary fibrosis. ${ }^{14,15}$ Many studies suggest that a comprehensive set of signaling pathways and proteins that contribute to the pathogenesis of pulmonary fibrosis can be inhibited by targeting VEGF/VEGFR2 signaling pathway. ${ }^{16,17}$ Our experiment has suggested that FXR could inhibit the progress of PF through inhibiting the migration of PMVECs, decreasing the angiogenesis, and reducing the expression of VEGF and VEGFR2.

PI3K can be activated by various cytokines in vivo, such as VEGF, transforming growth factor $\beta$ (TGF- $\beta$ ), connective tissue growth factor and so on. They all can mediate the activation of $\mathrm{PI} 3 \mathrm{~K} /$ protein kinase $\mathrm{B}$ (Akt) signaling pathway, leading to the fibroblasts' resistance to 


\section{VEGF}

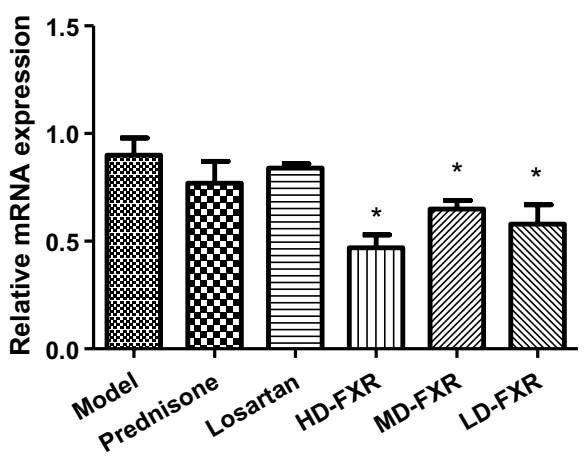

(a)
VEGFR2

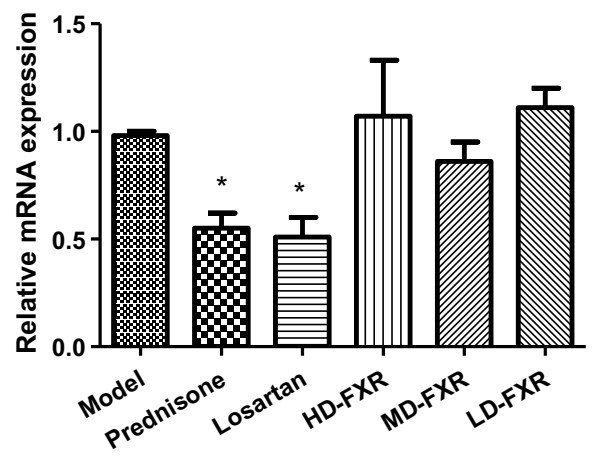

(b)

Figure 5. FXR inhibited the mRNA expression of VEGF and VEGFR2 in PMVECs. RT-PCR detection of relative expression of VEGF and VEGFR2 mRNA in six groups cultured PMVECs: (a) Analysis of the relative mRNA expression reveals that FXR can reduce the mRNA expression of VEGF significantly better than others. (b) losartan and prednisone can decrease the RNA expression of VEGFR, and have more advantage than FXR. Results are expressed as the means \pm SD. Notes: ${ }^{*} P<0.05$, Compared with model group. Model: model group, Prednisone: prednisone group, Losartan: losartan group, HD-FXR: high-dose of FXR group, MD-FXR: mid-dose of FXR group, LD-FXR: low-dose of FXR group.

PI3K

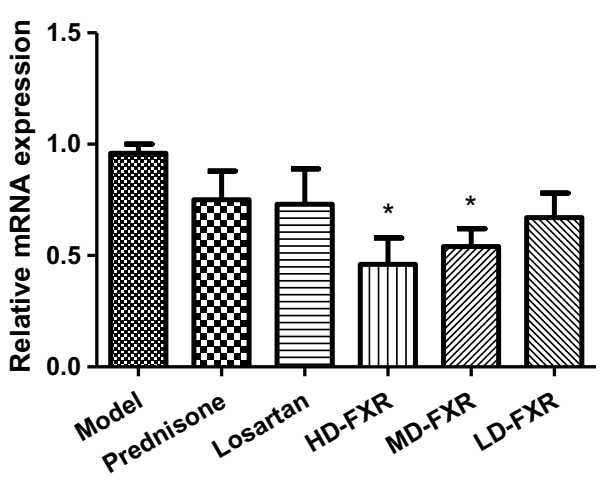

(a)
P38MAPK

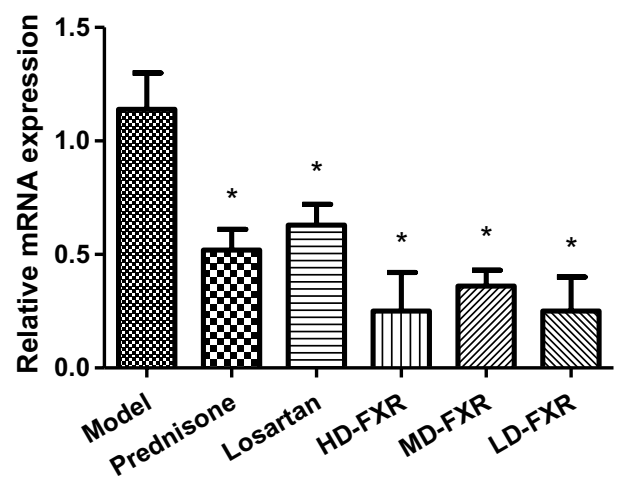

(b)

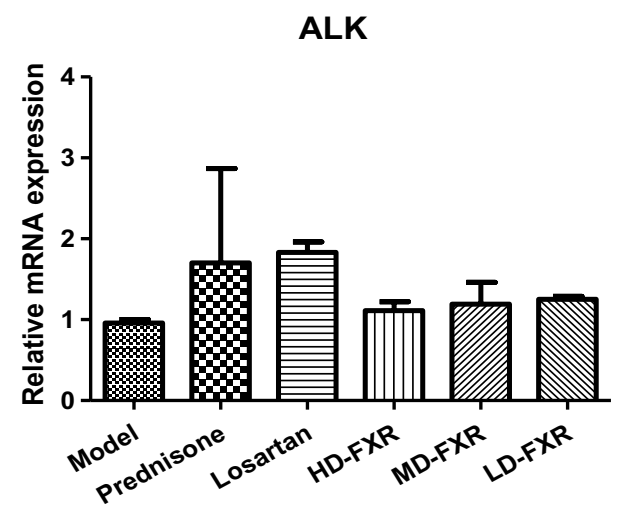

(c)

Figure 6. FXR inhibited the mRNA expression of PI3K, P38MAPK and ALK in PMVECs. RT-PCR detection of relative expression of PI3K, P38MAPK and ALK mRNA in six groups cultured PMVECs: (a) Analysis of the relative mRNA expression reveals that FXR can reduce the mRNA expression of PI3K significantly better than others. (b) FXR can down-regulate the RNA expression of P38MAPK significantly better than losartan and prednisone. (c) The mRNA expression of ALK in FXR, losartan and prednisone groups are up-regulated than model group, but high-dose of FXR can inhibit the upward trend. Results are expressed as the means $\pm \mathrm{SD}$.

Notes: $* P<0.05$, Compared with model group. Model: model group, Prednisone: prednisone group, Losartan: losartan group, HD-FXR: high-dose of FXR group, MD-FXR: mid-dose of FXR group, LD-FXR: low-dose of FXR group. 
apoptosis. More relative research was conducted in the tumor. ${ }^{14,18}$ P38MAPK pathway can be activated by VEGF and VEGFR. The activated P38MAPK can promote epithelial mesenchymal transition, participate in inflammatory cells infiltration, promote fibroblasts proliferation and differentiation and extracellular matrix deposition, leading to the development of pulmonary interstitial fibrosis. ${ }^{15,19,20}$ ALK is a type I receptor of the TGF- $\beta$ receptor (T $\beta$ R). The VEGF and VEGFR can activate ALK, and the activated ALK can promote the synthesis and deposition of collagen and inhibit the degradation of extracellular matrix, so that aggravate the progress of pulmonary fibrosis. ${ }^{21,22}$ Our experiment could not be a good proof for it, may be due to sample quality or other reasons, so we need to study further.

We believe that the traditional Chinese medicine pathogenesis of idiopathic pulmonary fibrosis is due to phlegm and blood stasis obstructing the lung, and deficiency of lung and kidney. ${ }^{23,24}$ FXR is the effective prescription of idiopathic pulmonary fibrosis summarized from clinical treatments, which has the benefits of replenishing and restoring lung and kidney, dissipating phlegm and eliminating blood stasis, and promoting blood circulation for removing obstruction in collaterals. The previous studies of animal experiments showed that FXR could play its function of anti-rat pulmonary fibrosis, anti-matrix remodeling, anti-coagulation ${ }^{25,26}$ by inhibiting the mRNA expression of plasminogen activator Inhibitor- 1 and cell adhesion molecule- $1 .{ }^{27}$ The results of this study suggest that FXR plays an important role in the process of inhabiting pulmonary fibrosis, may be by targeting PMVECs, VEGF/VEGFR2 signaling pathway, and the mRNA expression of PI3K, P38 MAPK and ALK.

\section{Conflict of Interest}

The authors declare that they have no conflict of interest.

\section{Acknowledgments}

This study received the help from department of pathology, Dongfang Hospital Affiliated to Beijing University of Chinese Medicine. This work is supported by the Natural Science Foundation of China (Study of Feixian Recipe Inhibit Angiogenesis and VEGF/VEGFR Signaling Pathway in Pulmonary Fibrosis Rats, No. 81273696).

\section{References}

1. Katzenstein AL, Myers JL. Idiopathic pulmonary fibrosis: Clinical relevance of pathologic classification. Am J Respir Crit Care Med 1998;157(1):1301-15.

2. Chambers RC, Mercer PF. Mechanisms of alveolar epithelial injury, repair, and fibrosis. Ann Am Thorac Soc 2015;12 (Supplement 1):S16-20.
3. Yan LF, Wei YN, Nan HY, et al. Proliferative phenotype of pulmonary microvascular endothelial cells plays a critical role in the overexpression of CTGF in the bleomycin-injured rat Exp Toxicol Pathol 2014;66(1):61-71.

4. Yin Q, Nan HY, Zhang WH, et al. Pulmonary microvascular endothelial cells from bleomycin-induced rats promote the transformation and collagen synthesis of fibroblasts. J Cell Physiol 2011;226(8):2091-102.

5. Yin Q, Nan H, Yan L, et al. Alteration of tight junctions in pulmonary microvascular endothelial cells in bleomycintreated rats. Exp Toxicol Pathol 2012;64(1-2):81-91.

6. Iyer AK, Ramesh V, Castro CA et al. Nitric oxide mediates bleomycin-induced angiogenesis and pulmonary fibrosis via regulation of VEGF. J Cell Biochem 2015;116(11):2484-93.

7. Mammoto T, Jiang A, Jiang E, Mammoto A. Role of twist1 phosphorylation in angiogenesis and pulmonary fibrosis. Am J Respir Cell Mol Biol 2016;55(5):633.

8. Smadja DM, Mauge L, Nunes H, et al. Imbalance of circulating endothelial cells and progenitors in idiopathic pulmonary fibrosis. Angiogenesis 2013;16(1):147-57.

9. Voelkel NF, Douglas IS, Nicolls M. Angiogenesis in chronic lung disease. Chest 2007;131(3):874.

10. Rahman HNA, Wu H, Dong Y, et al. Selective targeting of a novel epsin-VEGFR2 interaction promotes VEGF-mediated angiogenesis. Circ Res 2016;118(6):957.

11. Cui H, Wang Y, Huang H, et al. GPR126 protein regulates developmental and pathological angiogenesis through modulation of VEGFR2 receptor signaling. J Biol Chem 2014;289 (50):34871-85.

12. Richeldi L, Du BR, Raghu G, et al. Efficacy and safety of nintedanib in idiopathic pulmonary fibrosis. N Engl J Med 2014;370(22):2071.

13. Magro CM, Allen J, Popeharman A, et al. The role of microvascular injury in the evolution of idiopathic pulmonary fibrosis. Am J Clin Pathol 2003;119(4):556-67.

14. Gyori D, Chessa T, Hawkins P, et al. Class (I) Phosphoinositide 3-kinases in the tumor microenvironment. Cancers 2017;9(3):24

15. Molkentin JD, Bugg D, Ghearing N, et al. Fibroblast-specific genetic manipulation of p38 MAPK in vivo reveals its central regulatory role in fibrosis. Circulation 2017;136(6):549-61. doi: 10.1161/116.026238.

16. Venkatadri R, Iyer AKV, Ramesh V, et al. MnTBAP inhibits bleomycin-induced pulmonary fibrosis by regulating VEGF and Wnt signaling. J Cell Physiol 2016;232(3):506-16.

17. Kulkarni YM, Dutta S, Iyer AKV, et al. A proteomics approach to identifying key protein targets involved in VEGF inhibitor mediated attenuation of bleomycin-induced pulmonary fibrosis. Proteomics 2016;16(1):33.

18. Bi YL, Mi PY, Zhao SJ, et al. Salinomycin exhibits anti-angiogenic activity against human glioma in vitro and in vivo by suppressing the VEGF-VEGFR2-AKT/FAK signaling axis. Int $\mathrm{J}$ Mol Med 2017;39(5):1255-61.

19. Nimlamool W, Andrews RM, Falk MM. Connexin43 phosphorylation by PKC and MAPK signals VEGF-mediated gap junction internalization. Mol Biol Cell 2015;26(15):2755-68.

20. Guo L, Xu JM, Liu L, et al. Hypoxia-induced epithelialmesenchymal transition is involved in bleomycin-induced lung fibrosis. Biomed Res Int 2015:232791. doi: 10.1155/ 2015/232791

21. Mishra A, Stueckle TA, Mercer RR, et al. Identification of TGFbeta receptor-1 as a key regulator of carbon nanotube-induced 
fibrogenesis. Am J Physiol Lung Cell Mol Physiol 2015;309(8): L821-33.

22. Koh RY, Lim CL, Uhal BD, et al. Inhibition of transforming growth factor-beta via the activin receptor-like kinase-5 inhibitor attenuates pulmonary fibrosis. Mol Med Rep 2015;11(5):3808-13.

23. Jiang LD, Zhang XM, Xiao PX. Etiology and pathogenesis of idiopathic pulmonary interstitial fibrosis. Chin J Tradit Chin Med Pharm 2008(11):984-6.

24. Zhang XM, Jiang LD, Zhang W, et al. Influences of Feixian formula on PDGF, ET and TXB-2 in rats with pulmonary interstitial fibrosis induced by bleomycin. J Beijing Univ Tradit Chin Med. 2009;32(9):611-3.
25. Zhang XM, Jiang LD, Yin T, et al. The effect of feixian fang treating the idiopathic pulmonary fibrosis patients in MMPs and TIMPs. Chin J Tradit Chin Med Pharm 2009 (6):739-41.

26. Zhang X, Jiang L, Zhang W, et al. Effect of Feixian recipe on laminin, collagen I and III in rats with pulmonary fibrosis induced by bleomycin. Front Med 2008;2(3):314-6.

27. Liu Z, Zhang XM, Qin $\mathrm{H}$, et al. Feixianfang extract interfering vascular cell adhesion molecule 1 and plasminogen activator inhibitor-1 mRNA expression in rat model of pulmonary fibrosis. Chin J Exp Tradit Med Formulae 2015;21(7):132-5. 